\title{
The Impact of Self-Reported Recurrent Headache on Absenteeism and Presenteeism at Work Among Finnish Municipal Female Employees
}

This article was published in the following Dove Press journal: Journal of Pain Research

\author{
Kirsi Malmberg-Ceder ${ }^{1}$ \\ Tiina Vuorio ${ }^{2}$ \\ Päivi E Korhonen ${ }^{2}$ \\ Hannu Kautiainen ${ }^{3}$ \\ Seppo Soinila ${ }^{4}$ \\ Maija Haanpää ${ }^{5}$ \\ 'Department of Clinical Neurosciences, \\ University of Turku, Turku, Finland; \\ ${ }^{2}$ Department of General Practice, \\ University of Turku and Turku University \\ Hospital, Turku, Finland; ${ }^{3}$ Unit of Primary \\ Health Care, Kuopio University Hospital, \\ Kuopio, Finland; ${ }^{4}$ Department of Clinical \\ Neurosciences, University of Turku and \\ Turku University Hospital, Turku, Finland; \\ ${ }^{5}$ Mutual Insurance Company IImarinen, \\ Helsinki, Finland
}

\begin{abstract}
Purpose: The aim of this cross-sectional, observational study was to determine the impact of self-reported headache on absenteeism and presenteeism in a female working-age population.
\end{abstract}

Subjects and Methods: The study population consisted of 594 Finnish female municipal employees, who answered self-administered questionnaires including sociodemographic, lifestyle, health, and work-related data. Sickness absence days were obtained from the official records of the employer. Headache recurrence was defined by asking whether headache was occasional or recurrent. Headache impact was measured by the HIT-6.

Results: In our study, 456 (77\%) females had headache, and headache was recurrent in 178 $(39 \%)$. The self-reported recurrence of headache was related to age, AUDIT-C, health-rated quality-of-life, self-rated work ability, depressive symptoms, and work stress ( $P$ for linearity $<0.001$ ). They also had more depressive symptoms and work stress ( $P$ for linearity $<0.001$ ). Mental work load was highest in those with recurrent headache $(P=0.042)$, and work engagement was highest in those without headache $(P=0.038)$. There was no statistically significant difference in absenteeism days between the headache groups when adjusted with confounding variables. Presenteeism was associated with the recurrence of headache ( $P$ for linearity $<0.001)$. Presenteeism and the HIT-6 score were significantly associated in the recurrent headache group $(P=0.009)$.

Conclusion: Headache was not related to absenteeism, but the self-reported recurrence of headache was clearly associated with presenteeism in this female working-age population.

Keywords: work ability, sick leave, Headache Impact Test 6, HIT-6, lost productivity

\section{Introduction}

Headache is one of the most common disorders of the nervous system; its global lifetime prevalence is $66 \%{ }^{1-3}$ Headache is associated with substantial disability. ${ }^{4}$ Numerous earlier studies have shown that frequent or chronic headache impairs health-related quality-of-life. ${ }^{5,6}$ Burden of headache is present also during interictal periods in patients with episodic headache. ${ }^{7}$ Similarly to other pain conditions headache is more prevalent in females, and also comorbidity between pain conditions and mental and somatic problems is higher in women than in men. ${ }^{8}$ Comorbid conditions, for example other physical diseases or mental disorders, are often the major explanation for role disability in the headache population. ${ }^{9}$

The presence of a chronic illness has been shown to be a strong risk factor for sickness absence from work. ${ }^{10}$ Illness is also a cause of decreased productivity
Correspondence: Kirsi Malmberg-Ceder Email kirmal@utu.fi
Journal of Pain Research 2020:13 2135-2142

2135 
while working, a state called presenteeism, which in turn seems to lead to absenteeism. ${ }^{11,12}$ It is widely known that headache, especially severe migraine and chronic headache, causes absenteeism, and the socio-economic burden of headache for the individual and for society is substantial. ${ }^{1,13-16}$ Headache prevalence is high, especially among working-age females, and headache has a negative impact on different aspects of life. ${ }^{17}$ Headache severity is more relevant than the headache diagnosis regarding work ability. ${ }^{18}$ Work-related psychosocial risk factors such as role conflict, low social climate, and bullying may provoke headache. ${ }^{19}$ To our knowledge, only one study has evaluated work engagement in a headache population. ${ }^{20}$ It showed that job demands and job resources are important for work ability in employees with chronic headache.

The aim of this cross-sectional, observational study was to determine the impact of the self-reported recurrent headache on absenteeism and presenteeism among a female working-age population.

\section{Subjects and Methods Study Population}

This report is based on a longitudinal cohort study comprising employees of the city of Pori (Southwestern Finland). The study material was collected in 2014-2015 as part of the PORTAAT (PORi To Aid Against Threats) study. ${ }^{21}$ The managers of the work units sent the invitation and study information letters by e-mail to the employees. No exclusion criteria were applied. Subjects from 10 work units were enrolled and occupations included were librarians, museum employees, groundkeepers, computer workers, social workers, nurses, physicians, administrative officials, and general office staff. All study subjects were informed in an appointment with the study nurse and consented to the study.

For the present analyses, we included 594 females who completed the follow-up visit in 2015, and who had completed the work-related questionnaires and the headache questionnaire. The study subjects answered the question "Have you had headache during the past year?" (yes/no). If the subject had suffered from headache, the recurrence of it was assessed with the question "Has your headache been recurrent?" (yes/no). Accordingly, the headache population was categorized as having occasional or recurrent self-reported headache. Both headache groups filled in the HIT-6 questionnaire.

\section{Sociodemographic, Lifestyle, and Health-Related Factors}

Demographic, lifestyle, and health data were collected using self-administered questionnaires. Information was gathered about years of education, financial satisfaction (with the question "Do you have save on expenditures?"; "yes" or "no"), marital status ("cohabiting or not"), smoking ("current smoker or non-smoking (having never smoked or having stopped smoking $>12$ months ago"), alcohol consumption (the 3-item Alcohol Use Disorders Identification Test, AUDIT-C), and quality of sleep ("good" or "not good"). ${ }^{22}$ Leisure-time physical activity (LTPA) was assessed by asking for the recurrence and duration of physical activities during a typical week. LTPA was considered high if activity was $\geq 30$ minutes at a time for four or more times a week; moderate if $\geq 30$ minutes at a time for two to three times a week and low for $\geq 30$ minutes at a time for a maximum of once a week. Health-related quality-of-life was assessed with the EQ-5D questionnaire. $^{23}$

The subjects self-rated their mood using the Major Depression Inventory (MDI) questionnaire. MDI measures depressive symptoms during the past 2 weeks. ${ }^{24}$ MDI consist of ten items, each evaluated on a Likert-type scale from $0=$ never to $5=$ all the time. The total score of the MDI ranges from 0 to 50 . The higher the score, the more severe is the subject's depression. Optimal cut-off score for major (moderate-to-severe) depression is 26. Height and weight of the participants were measured by the study nurse. Body mass index (BMI) was calculated as weight $(\mathrm{kg})$ divided by the square of height $\left(\mathrm{m}^{2}\right)$. Medical records and self-administered questionnaires served the source of information on the subjects' chronic diseases and regular medications. A study subject was considered to have diabetes, malignancies, musculoskeletal, cardiovascular, psychiatric, pulmonary, gastroenterological, or neurological diseases, if the disease was diagnosed by a physician and/or she used appropriate medication.

\section{Headache Impact Test (HIT-6)}

To assess the impact of headache the HIT-6 was used. It is a self-administered questionnaire presenting three questions concerning headache during the past 4-week period and a further three questions about headaches with no time limit. ${ }^{25}$ Each of the HIT-6 items is scored for frequency using the scale never $=6$, rarely $=8$, sometimes $=10$, very often $=11$ and always $=13$, the total score ranging from 36 to 
78. Based on the total HIT-6 score, the subjects can be categorized into four groups according to the impact of headache: little or no impact $(<50)$, some impact $(50-55)$, substantial impact (56-59), and very severe impact $(\geq 60){ }^{26}$ The study subjects filled in the new Finnish version of the HIT-6 questionnaire, which was produced by the forward-backward translation process. A new Finnish translation was done because of problems in the earlier Finnish version of HIT$6 .^{27}$ The new Finnish translation was performed without the approval of OptumInsight Life Sciences (QualityMetrics), but a retroactive license has since been issued.

\section{Work-Related Factors Work Engagement}

The Utrecht Work Engagement Scale (UWES-9) measures the work engagement. ${ }^{28}$ UWES-9 consists of three subscales, which focus on vigor, dedication, and absorption. Sub-scales were rated on a 7-point Likert scale ranging from 0 (strongly disagree) to 6 (strongly agree). The overall work engagement score was obtained by summing up all items and dividing by the number of items in each scale. The overall work engagement is higher when the item is rated higher. In our study work engagement tertiles were $1 ;<4.5,2 ; 4.6-5.2$, and $3 ;>5.3$.

\section{Work Ability}

To evaluate the work ability, a single question was presented to the subjects: "What is your current work ability compared to your lifetime best?". This is the first item in the widely used Work Ability Index (WAI), defined as the Work Ability Score (WAS). ${ }^{29}$ WAS is obtained using a $0-10$ response scale, 0 denoting complete inability to work and 10 indicating "work ability at its best". Reference values for WAS transformed to WAI; poor (0-5 points), moderate (6-7), good (8-9), and excellent (10). WAS and WAI are strongly associated and are accurate indicators of work ability. ${ }^{30}$

\section{Physical and Mental Workload}

Physical workload was assessed with the question "How strenuous is your work physically?" and mental workload with the question "How strenuous is your work mentally?". Answers were given with a $100 \mathrm{~mm}$ long visual analog scale (VAS) and with a 0-100 response scale $(0=$ very light to $100=$ very hard $)$.

\section{Work Stress}

The Bergen Burnout Indicator (BBI-15) was used to evaluate work stress. ${ }^{31}$ BBI-15 measures occupational burnout using 15 questions and the answers are given using Likerttype scales from 1 to $6(1=$ completely disagree to $6=\mathrm{com}$ pletely agree), that are summed up to score from 15 to 90 , a high score indicating high levels of work stress.

\section{Daytime Work and Absenteeism Records}

The data concerning daytime or shift-work and the count of sickness absence days during the 2-year period of January 1, 2014-December 31, 2015 were obtained from the official records of the employer (the city of Pori). The mean of sick leave days of 2014 and 2015 was used.

\section{Presenteeism}

Presenteeism at work was assessed with a question and a $100 \mathrm{~mm}$ long visual analog scale with advice for use: "If you had work days during the past month, evaluate how much your health problems have affected your work performance while working"(from $0=$ no problems to $100=$ completely hindered my work performance).

\section{Ethics Approval and Consent to Participate}

The Ethics Committee of the Hospital District of Southwestern Finland reviewed and approved the study protocol and consent forms. The written informed consent was given by all participants.

\section{Statistical Methods}

The statistical significance for the unadjusted hypothesis of linearity across categories (quartiles) of headache and characteristics of the study participants were evaluated using the Cochran-Armitage test for trend, analysis of variance (ANOVA), and logistic (ordinal) models with an appropriate contrast. Adjusted relationships between categories of headache and absenteeism days or presenteeism were analyzed using generalized linear models with appropriate distribution and link function. In the case of violation of the assumptions (eg, non-normality), a bootstrap-type test was used. The normality of variables was evaluated graphically and using the Shapiro-Wilk $W$-test. Stata 16.0 (StataCorp LP, College Station, TX, USA) was used for the analysis.

\section{Results}

The study population consisted of 594 female employees, of whom 456 (77\%) had headache symptoms during the last year. Headache was recurrent in 178 (39\%) subjects. The characteristics of the subjects according to the selfreported headache recurrence are shown in Table 1. 
Table I Characteristics of Study Subjects According to Categories of Self-Reported Headache Recurrence

\begin{tabular}{|c|c|c|c|c|}
\hline & \multicolumn{3}{|c|}{ Self-Reported Recurrence of Headache } & \multirow[t]{2}{*}{$P$-value for Linearity } \\
\hline & No $(N=138)$ & Occasional $(\mathrm{N}=\mathbf{2 7 8})$ & Recurrent $(\mathrm{N}=178)$ & \\
\hline \multicolumn{5}{|l|}{ Sociodemographic factors } \\
\hline Age, years, mean (SD) & $5 \mathrm{I}(9)$ & $49(10)$ & $47(10)$ & $<0.001$ \\
\hline Education years, mean $(S D)$ & $13.6(2.2)$ & $13.8(2.1)$ & $13.8(2.1)$ & 0.46 \\
\hline Financial satisfaction, n (\%) & $105(76)$ & $203(73)$ & $125(70)$ & 0.24 \\
\hline Cohabiting, n (\%) & $109(79)$ & $224(8 I)$ & $146(82)$ & 0.50 \\
\hline \multicolumn{5}{|l|}{ Lifestyle factors } \\
\hline Smoking, n (\%) & $14(10)$ & $23(8)$ & $16(9)$ & 0.77 \\
\hline AUDIT-C, mean (SD) & $3.1(1.5)$ & $2.8(1.5)$ & $2.5(1.6)$ & $<0.001$ \\
\hline Good quality of sleep, n (\%) & III (80) & $212(76)$ & $132(74)$ & 0.20 \\
\hline Leisure time physical activity, n (\%) & & & & 0.44 \\
\hline Low & $25(18)$ & $56(20)$ & $39(22)$ & \\
\hline Moderate & $64(46)$ & $120(43)$ & $80(45)$ & \\
\hline High & $49(36)$ & $102(37)$ & $59(33)$ & \\
\hline \multicolumn{5}{|l|}{ Health-related factors } \\
\hline Quality-of-life (EQ-5D), mean (SD) & $0.90(0.12)$ & $0.88(0.12)$ & $0.81(0.17)$ & $<0.001$ \\
\hline Depressive symptoms (MDI), mean (SD) & $4.1(5.8)$ & $5.1(5.8)$ & $6.1(5.4)$ & 0.001 \\
\hline $\mathrm{BMI}, \mathrm{kg} / \mathrm{m}^{2}$, mean $(\mathrm{SD})$ & $26.2(4.3)$ & $26.6(4.8)$ & $27.2(5.4)$ & 0.002 \\
\hline Number of chronic illnesses, mean (SD) & I.I (I.I) & $\mathrm{I} .0(\mathrm{I} . \mathrm{I})$ & $1.3(1.4)$ & 0.079 \\
\hline Musculoskeletal & $28(20)$ & $55(20)$ & $43(24)$ & 0.37 \\
\hline Cardiovascular & $32(23)$ & $47(17)$ & $34(19)$ & 0.42 \\
\hline Mental & $3(2)$ & II (4) & $10(6)$ & 0.12 \\
\hline Pulmonary & $9(7)$ & $22(8)$ & $16(9)$ & 0.42 \\
\hline Gastroenterological & $10(7)$ & $21(8)$ & $18(10)$ & 0.33 \\
\hline Neurological & $4(3)$ & $9(3)$ & $4(2)$ & 0.70 \\
\hline Diabetes & $10(7)$ & $6(2)$ & $6(3)$ & 0.10 \\
\hline Malignancy & $2(1)$ & $3(4)$ & $5(3)$ & 0.56 \\
\hline \multicolumn{5}{|l|}{ Work-related factors } \\
\hline Work engagement (UWES-9 score), mean (SD) & $5.0(0.9)$ & $4.8(1.0)$ & $4.8(0.9)$ & 0.038 \\
\hline Work ability score, NRS, mean (SD) & $8.6(1.3)$ & $8.5(1.1)$ & $8.1(1.3)$ & $<0.001$ \\
\hline Physical workload, mm, mean (SD) & $33(28)$ & $27(26)$ & $30(27)$ & 0.54 \\
\hline Mental workload, mm, mean (SD) & $59(21)$ & $57(22)$ & $63(22)$ & 0.042 \\
\hline Work stress (BBI-I5), mean (SD) & $29(\mathrm{II})$ & $32(10)$ & $33(11)$ & $<0.001$ \\
\hline Daytime work, n (\%) & $94(68)$ & $201(72)$ & $132(74)$ & 0.25 \\
\hline
\end{tabular}

Abbreviations: AUDIT-C, Alcohol Use Disorders Identification Test; EQ-5D, quality-of-life; MDI, Major Depression Inventory; BMI, body mass index; UWES-9, Utrecht Work Engagement Index; BBI, Bergen Burnout Indicator.

Females with an increasing level of headache were more likely to be younger, have higher BMI, lower AUDIT-C score, lower health-related quality-of-life, and lower work ability.

Recurrence of headache was related to age, AUDIT-C, health-related quality-of-life, self-rated work ability, depressive symptoms, and work stress $(P$ for linearity $<0.001$ ). Mental work load was highest in those with recurrent headache $(P=0.042)$, and work engagement was highest in those without headache $(P=0.038)$. There was no statistically significant difference in absenteeism days between the headache groups when adjusted with confounding variables. Presenteeism was associated with the recurrence of headache ( $P$ for linearity $<0.001)$. Presenteeism and the HIT-6 score were significantly associated in the recurrent headache group $(P=0.009)$.

The mean number of absenteeism days and the mean level of presenteeism are presented in Table 2, both as crude results (model I) and after adjustments (models II-IV). The number of absenteeism days was highest in the recurrent headache group both as crude results (model I) and after adjustments (models II-IV), but the relation was statistically significant 
Table 2 The Mean Number of Absenteeism Days during Years 2014-2015, and the Mean Level of Presenteeism (VAS 0-100) According to the Self-Reported Headache Recurrence Categories

\begin{tabular}{|c|c|c|c|}
\hline \multirow[t]{2}{*}{ Headache Recurrence } & \multicolumn{2}{|l|}{ Absenteeism Days } & \multirow{2}{*}{$\begin{array}{l}\text { Presenteeism, VAS } \\
\text { Mean }(95 \% \mathrm{Cl})\end{array}$} \\
\hline & Mean $(95 \% \mathrm{Cl})$ & Ratio $(95 \% \mathrm{Cl})$ & \\
\hline \multicolumn{4}{|l|}{ Model I } \\
\hline No & $19.3(14.1-24.6)$ & I (Reference) & $10.7(7.2-14.1)$ \\
\hline Occasional & $19.0(15.4-22.6)$ & $0.98(0.70-1.37)$ & $13.3(10.8-15.7)$ \\
\hline Recurrent & $27.3(20.7-33.8)$ & $1.41(0.98-2.02)$ & $21.6(18.6-24.6)$ \\
\hline & $P$ for linearity $=0.047$ & & $P$ for linearity $<0.001$ \\
\hline \multicolumn{4}{|l|}{ Model II } \\
\hline No & I8.1 (13.3-22.9) & I (Reference) & $10.7(7.3-14.1)$ \\
\hline Occasional & $17.2(14.0-20.3)$ & $0.94(0.69-1.31)$ & $13.3(10.9-15.7)$ \\
\hline Recurrent & $25.5(19.6-31.5)$ & $1.41(0.99-2.00)$ & $21.3(18.3-24.3)$ \\
\hline & $P$ for linearity $=0.039$ & & $P$ for linearity $<0.001$ \\
\hline \multicolumn{4}{|l|}{ Model III } \\
\hline No & $18.3(13.3-23.2)$ & I (Reference) & $10.4(7.0-13.9)$ \\
\hline Occasional & $17.2(14.0-20.4)$ & $0.94(0.68-1.31)$ & $13.4(\mid 1.0-15.8)$ \\
\hline Recurrent & $24.9(18.9-30.8)$ & $1.36(0.94-2.97)$ & $21.5(18.5-24.6)$ \\
\hline & $P$ for linearity $=0.076$ & & $P$ for linearity $<0.001$ \\
\hline \multicolumn{4}{|l|}{ Model IV } \\
\hline No & $16.1(12.0-20.3)$ & I (Reference) & $12.2(9.0-15.4)$ \\
\hline Occasional & $17.0(13.9-20.0)$ & $1.05(0.77-1.44)$ & $13.4(\mid 1.1-15.6)$ \\
\hline \multirow[t]{2}{*}{ Recurrent } & $21.7(16.7-26.7)$ & $1.35(0.94-1.92)$ & $19.7(16.8-22.5)$ \\
\hline & $P$ for linearity $=0.089$ & & $P$ for linearity $<0.001$ \\
\hline
\end{tabular}

Notes: Model I crude; Model II adjusted for age, BMI, and education years; Model III adjusted model II+ smoking, AUDIT-C, and LTPA; Model IV adjusted model III+ MDI, $\mathrm{BBI}$, daytime work, and number of chronic illnesses.

Abbreviations: BMI, body mass index; AUDIT-C, Alcohol Use Disorders Identification Test; LTPA, leisure time physical activity; MDI, Major Depression Inventory; BBI, Bergen Burnout Indicator.

only in models I and II (ie, crude results and when adjusted for age, BMI, and education years). Presenteeism had a significant positive association with headache recurrence.

The number of absenteeism days and the level of presenteeism by HIT- 6 categories in the occasional and recurrent headache groups using model IV (adjusted for age, BMI, education years, smoking, AUDIT-c score, LTPA, MDI, $\mathrm{BBI}$, daytime work, and number of chronic illnesses) are presented in Figure 1. In the recurrent headache group categories of HIT-6 were positively associated with presenteeism $(P=0.009)$ but not with absenteeism $(P=0.36)$. In the occasional headache group neither absenteeism $(P=0.29)$ nor presenteeism $(P=0.71)$ was associated with the HIT-6 categories.

\section{Discussion}

The main finding of this study consisting of female municipal employees was that self-reported recurrent headache is associated with presenteeism but not with absenteeism, even after adjustments. In the recurrent headache group, but not in the occasional headache group, presenteeism was significantly associated with the burden of headache.

To our knowledge, our study is the first to assess the association of the HIT-6 score with presenteeism in a working aged female population. Presenteeism was clearly more evident among the females suffering from recurrent headache than among those with occasional or no headache. This highlights the importance of recognizing the patients with recurrent symptoms. The HIT-6 is easy to use in everyday clinical practice. Thus, we encourage using the questionnaire to find the patients with high burden of headache and increased risk for presenteeism. It has been shown that presenteeism is a risk factor for absenteeism and the economic costs of presenteeism have been suggested to even exceed those of corresponding absenteeism ${ }^{11,12,32,33}$. Although often multifactorial, recurrent headache can usually be treated efficiently with low cost procedures when noticed early (eg, good migraine acute treatment and prevention, relieving muscle tension, giving lifestyle guidance and psychological support during difficult life events). 
There was no association between HIT-6 score and absenteeism in this study, which may be explained by the characteristics of the study population. Absenteeism was highest in the recurrent headache group compared to occasional headache or no headache groups with no or minor adjustments, but when adjusted by several lifestyle and health-related variables absenteeism did not significantly correlate to the headache recurrence. According to several earlier studies headache is associated with absenteeism, but comorbidities, especially mental disorders, have a substantial role in absenteeism in a headache population. ${ }^{9,15}$ Our study subjects were quite healthy municipal employees with only mild mental symptoms, which presumably explains the low absenteeism in this study.

The headache prevalence in our study was approximately of the same magnitude as in earlier Scandinavian studies. $^{2}$ The characteristics of headache are also of concern when speculating the reason for absenteeism. Decreased work ability is clearly shown in those with migraine and also in frequent or chronic headache populations. ${ }^{13,16,17,34-36}$ Population-based studies have shown that in a headache population, especially in episodic headache populations, presenteeism is more substantial than absenteeism. ${ }^{37,38}$ It has been estimated that presenteeism is responsible for two thirds and absenteeism for one third of migraine-related indirect costs. ${ }^{36}$ If headache is mild or moderate or is rapidly alleviated by acute medication (which is the case in most migraine patients), the subject may consider sick-leave excessive and goes to work. Headache-related absence is known to be stigmatized, which may also explain avoidance of sick leave days due to headache. ${ }^{39}$

The strength of the present study is a well-characterized and relatively large cohort of employees comprising a study population with relatively homogeneous cultural background. The participants receive equitable salaries, their working conditions are regulated by the same collective agreement, their employment status is stable, and they share a uniform occupational healthcare system, even though they represent different work units and widely varying tasks. Only female employees were included in this substudy, because the total number of males in the PORTAAT study was low and hence the homogeneity of the study population was increased. The questionnaires which were used in this study are valid and reliable for measuring workrelated factors and the impact of headache. The data on sick-leave days were gathered from the employer, ie, from an official register. The comprehensive adjustments were made, because besides illnesses of the employee, numerous other sociodemographic, health-related, and work-related factors have been recognized as risk factors for absenteeism and presenteeism at work. ${ }^{40,41}$

The major limitation of this study is the cross-sectional design, which does not allow us to draw any causal conclusions. Another limitation is the lack of exact headache diagnosis and headache frequency due to the study design. This study observes the headache as a symptom, and the burden of the pain is measured by the HIT- 6 which is not a diagnose-specific questionnaire. It is likely that most females in the recurrent headache group have a headache disease, such as migraine, whereas in the occasional headache group the symptom may be a random sign of infection, hypertension, lack of sleep, etc. It is also liable that females with higher HIT-6 score have a headache diagnosis, eg, migraine or chronic headache. There might also be some overlapping between the headache groups, for example females with only minor migraine symptoms may have been categorized in the occasional headache group. Nonetheless, our aim was to study the association between reduced work ability (both absenteeism and presenteeism) and the self-reported recurrence of headache, regardless of
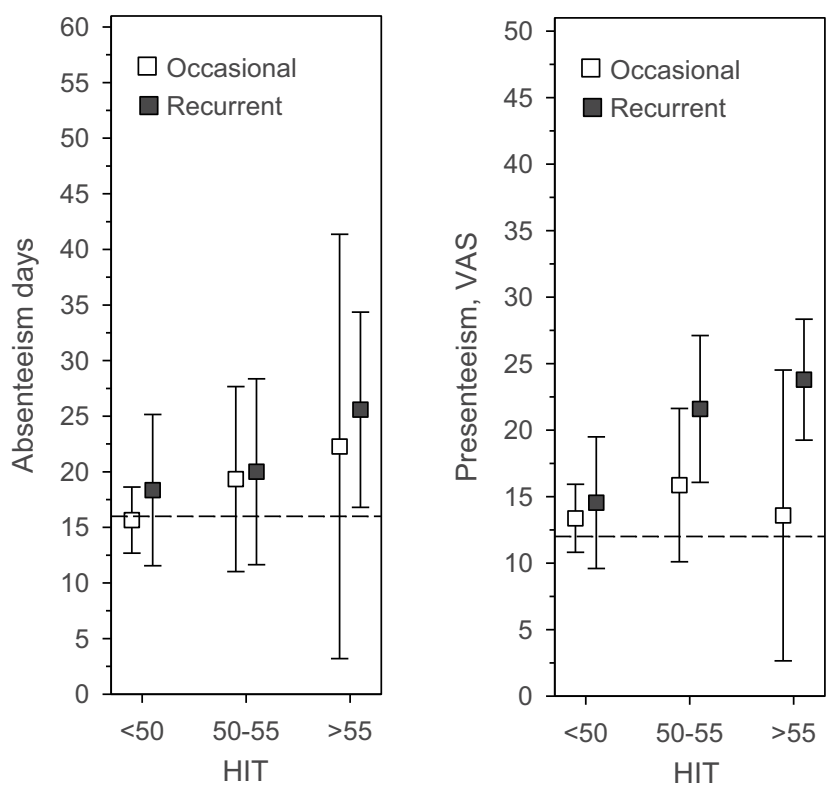

Figure I The mean number of absenteeism days during years 20I4-20I5 and the level of presenteeism (VAS 0-100) by HIT-6 categories in the self-reported occasional and recurrent headache groups. Error bars are for $95 \%$ confidence intervals. Dashed lines indicate mean values of absenteeism days and presenteeism in the whole study population. Data was adjusted using model IV (adjusted for age, BMI, education years, smoking, AUDIT-C score, LTPA, MDI score, BBI score, daytime work, and number of chronic illnesses).

Abbreviations: BMI, body mass index; AUDIT-C, Alcohol Use Disorders Identification Test; LTPA, leisure time physical activity; MDI, Major Depression Inventory; $\mathrm{BBI}$, Bergen Burnout Indicator. 
the exact diagnosis in a working-aged female population, so missing data do not affect the results or conclusions. There are only a few women with high headache burden (HIT-6 score over 55) in the occasional headache group, a phenomenon seen as wide confidential intervals in Figure 1. Lastly, because this study consists of only female subjects, the results cannot be generalized to the male population.

\section{Conclusion}

This study showed that in female municipal employees self-reported recurrent headache was associated with impaired productivity at work, mostly by presenteeism and with low absenteeism. Increased headache burden measured by the HIT- 6 was related to presenteeism, but not to absenteeism.

\section{Abbreviations}

AUDIT-C, Alcohol Use Disorders Identification Test; BBI, Bergen Burnout Indicator; BMI, body mass index; EQ-5D, quality-of-life; LTPA, leisure time physical activity; MDI, Major Depression Inventory; UWES-9, Utrecht Work Engagement; WAI, Work Ability Index; WAS, Work Ability Score.

\section{Disclosure}

We gratefully acknowledge the unrestricted research grants from the Mutual Insurance Company Etera and from the Finnish Cultural Foundation. Dr Maija Haanpää reports personal fees from Pfizer, outside the submitted work. The authors report no other conflicts of interest in this work.

\section{References}

1. Andlin-Sobocki P, Jönsson B, Wittchen HU, et al. Cost of disorders of the brain in Europe. Eur $J$ Neurol. 2005;12(Suppl 1):1-27. doi:10.1111/j.1468-1331.2005.01202.x

2. Stovner L, Hagen K, Jensen R, et al. The global burden of headache: a documentation of headache prevalence and disability worldwide. Cephalalgia. 2007;27(3):193-210. doi:10.1111/j.1468-2982.2007. 01288.x

3. Jensen R, Stovner LJ. Epidemiology and comorbidity of headache. Lancet Neurol. 2008;7(4):354-361. doi:10.1016/S1474-4422(08) 70062-0

4. Jensen R, Rasmussen BK. Burden of headache. Expert Rev Pharmacoecon Outcomes Res. 2004;4(3):353-359. doi:10.1586/ 14737167.4.3.353

5. Bigal ME, Bigal JM, Betti M, et al. Evaluation of the impact of migraine and episodic tension-type headache on the quality of life and performance of a university student population. Headache. 2001;41(7):710-719. doi:10.1046/j.1526-4610.2001.041007710.x
6. Michel P, Dartigues JF, Lindoulsi A, et al. Loss of productivity and quality of life in migraine sufferers among French workers: results from the GAZEL cohort. Headache. 1997;37(2):71-78. doi:10.1046/ j.1526-4610.1997.3702071.x

7. Lampl C, Thomas H, Stovner LJ, et al. Interictal burden attributable to episodic headache: findings from the Eurolight project. J Headache Pain. 2016;17:9. doi:10.1186/s10194-016-0599-8

8. Bingefors K, Isacson D. Epidemiology, co-morbidity, and impact on health-related quality of life of self-reported headache and musculoskeletal pain-a gender perspective. Eur J Pain. 2004;8(5):435-450. doi:10.1016/j.ejpain.2004.01.005

9. Saunders K, Merikangas K, Low NC, et al. Impact of comorbidity on headache-related disability. Neurology. 2008;70(7):538-547. doi:10.1212/01.wnl.0000297192.84581.21

10. Sundstrup E, Jakobsen MD, Mortensen OS, et al. Joint association of multimorbidity and work ability with risk of long-term sickness absence: a prospective cohort study with register follow-up. Scand J Work Environ Health. 2017;43(2):146-154. doi:10.5271/sjweh.3620

11. Aronsson G, Gustafsson K, Dallner M. Sick but yet at work. An empirical study of sickness presenteeism. J Epidemiol Community Health. 2000;54(7):502-509. doi:10.1136/jech.54.7.502

12. Skagen K, Collins AM. The consequences of sickness presenteeism on health and wellbeing over time: a systematic review. Soc Sci Med. 2016;161:169-177. doi:10.1016/j.socscimed.2016.06.005

13. Rasmussen BK, Jensen R, Olesen J. Impact of headache on sickness absence and utilisation of medical services: a Danish population study. J Epidemiol Community Health. 1992;46(4):443-446. doi:10. 1136/jech.46.4.443

14. Durham CF, Alden KR, Dalton JA, et al. Quality of life and productivity in nurses reporting migraine. Headache. 1998;38(6):427-435. doi:10.1046/j.1526-4610.1998.3806427.x

15. Michel P, Dartigues JF, Duru G, et al. Incremental absenteeism due to headaches in migraine: results from the Mig-Access French national cohort. Cephalalgia. 1999;19(5):503-510. doi:10.1046/j.1468-2982. 1999.019005503.x

16. Stewart WF, Wood GC, Manack A, et al. Employment and work impact of chronic migraine and episodic migraine. J Occup Environ Med. 2010;52(1):8-14. doi:10.1097/JOM.0b013e3181c1dc56

17. Allena M, Steiner TJ, Sances G, et al. C. Impact of headache disorders in Italy and the public-health and policy implications: a population-based study within the Eurolight Project. $J$ Headache Pain. 2015;16:100. doi:10.1186/s10194-015-0584-7

18. Pransky GS, Berndt E, Finkelstein SN, et al. Performance decrements resulting from illness in the workplace: the effect of headaches. J Occup Environ Med. 2005;47(1):34-40. doi:10.1097/01.jom. 0000150208.20117.34

19. Tynes T, Johannessen HA, Sterud T. Work-related psychosocial and organizational risk factors for headache: a 3-year follow-up study of the general working population in Norway. J Occup Environ Med. 2013;55(12):1436-1442. doi:10.1097/JOM.0b013e3182a7e678

20. van der Doef MP, Schelvis RMC. Relations between psychosocial job characteristics and work ability in employees with chronic headaches. J Occup Rehabil. 2019;29(1):119-127. doi:10.1007/s10926-0189769-7

21. Veromaa V, Kautiainen H, Saxen U, et al. Ideal cardiovascular health and psychosocial risk factors among Finnish female municipal workers. Scand J Public Health. 2017;45(1):50-56. doi:10.1177/ 1403494816677661

22. Bush K, Kivlahan DR, McDonell MB, et al. The AUDIT alcohol consumption questions (AUDIT-C): an effective brief screening test for problem drinking. Ambulatory care quality improvement project (ACQUIP). Alcohol use disorders identification test. Arch Intern Med. 1998;158(16):1789-1795. doi:10.1001/archinte.158.16.1789

23. Rabin R, de Charro F. EQ-5D: a measure of health status from the EuroQol Group. Ann Med. 2001;33(5):337-343. doi:10.3109/ 07853890109002087 
24. Bech P, Rasmussen NA, Olsen LR, et al. The sensitivity and specificity of the major depression inventory, using the present state examination as the index of diagnostic validity. $J$ Affect Disord. 2001;66(2-3):159-164. doi:10.1016/S0165-0327(00)00309-8

25. Kosinski M, Bayliss MS, Bjorner JB, et al. A six-item short-form survey for measuring headache impact: the HIT-6. Qual Life Res. 2003;Dec;12(8):963-974. doi:10.1023/A:1026119331193

26. Bayliss MS, Batenhorst AS. The HIT-6TM: A User's Guide. Lincoln, RI: QualityMetric; 2002.

27. Martin M, Blaisdell B, Kwong JW, et al. The short-form headache impact test (HIT-6) was psychometrically equivalent in nine languages. J Clin Epidemiol. 2004;57(12):1271-1278. doi:10.1016/ j.jclinepi.2004.05.004

28. Schaufeli WB, Salanova M, Gonzalez-Roma V, et al. The measurement of engagement and burnout: a confirmative analytic approach. J Happiness Stud. 2002;3(1):71-92. doi:10.1023/A:1015630930326

29. Tuomi K, Ilmarinen J, Jahkola A, et al. Work Ability Index. 2nd ed. Helsinki: Finnish Institute of Occupational Health; 1998.

30. Ahlstrom L, Grimby-Ekman A, Hagberg M, et al. The work ability index and single-item question: associations with sick leave, symptoms, and health - a prospective study of women on long-term sick leave. Scand J Work Environ Health. 2010;36(5):404-412. doi:10.5271/sjweh.2917

31. Näätänen P, Aro A, Matthiesen SB, et al. Bergen Burnout Indicator 15. Helsinki, Finland: Edita Publishing Oy; 2003. (in Finnish)

32. Stewart WF, Ricci JA, Chee E, Morganstein D. Lost productive work time costs from health conditions in the United States: results from the American Productivity Audit. J Occup Environ Med. 2003;45 (12):1234-1246. doi:10.1097/01.jom.0000099999.27348.78

33. Collins JJ, Baase CM, Sharda CE, et al. The assessment of chronic health conditions on work performance, absence, and total economic impact for employers. J Occup Environ Med. 2005;47(6):547-557. doi:10.1097/01.jom.0000166864.58664.29
34. Buse D, Manack A, Serrano D, et al. Headache impact of chronic and episodic migraine: results from the American Migraine Prevalence and Prevention study. Headache. 2012;52(1):3-17. doi:10.1111/ j.1526-4610.2011.02046.x

35. Burton WN, Conti DJ, Chen C-Y, Schultz AB, Edington DW. The economic burden of lost productivity due to migraine headache: a specific worksite analysis. J Occup Environ Med. 2002;44 (6):523-529. doi:10.1097/00043764-200206000-00013

36. Linde M, Gustavsson A, Stovner LJ, et al. The cost of headache disorders in Europe: the Eurolight project. Eur J Neurol. 2012;19 (5):703-711. doi:10.1111/j.1468-1331.2011.03612.x

37. Stewart WF, Wood GC, Razzaghi H, et al. Work impact of migraine headaches. J Occup Environ Med. 2008;50(7):736-745. doi:10.1097/ JOM.0b013e31818180cb

38. Hedenrud T, Löve J, Staland-Nyman C, et al. Frequent headache and work ability: a population-based study in Sweden. J Occup Environ Med. 2014;56(5):472-476. doi:10.1097/JOM.0000000000000156

39. Young WB, Park JE, Tian IX, et al. The stigma of migraine. PLoS One. 2013;8(1):e54074. doi:10.1371/journal.pone.0054074

40. Laaksonen M, Piha K, Martikainen P, et al. Health-related behaviours and sickness absence from work. Occup Environ Med. 2009;66 (12):840-847. doi:10.1136/oem.2008.039248

41. Slany C, Schütte S, Chastang JF, et al. Psychosocial work factors and long sickness absence in Europe. Int $J$ Occup Environ Health. 2014;20(1):16-25. doi:10.1179/2049396713Y.0000000048
Journal of Pain Research

\section{Publish your work in this journal}

The Journal of Pain Research is an international, peer reviewed, open access, online journal that welcomes laboratory and clinical findings in the fields of pain research and the prevention and management of pain. Original research, reviews, symposium reports, hypothesis formation and commentaries are all considered for publication. The manuscript management system is completely online and includes a very quick and fair peer-review system, which is all easy to use. Visit http:/ www.dovepress.com/testimonials.php to read real quotes from published authors. 